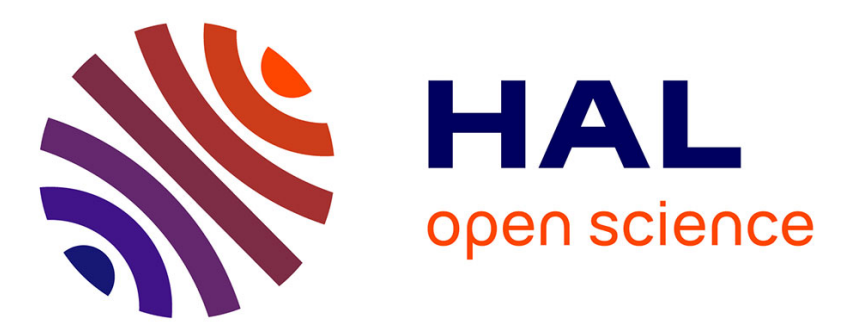

\title{
Hyper-parameter optimization tools comparison for Multiple Object Tracking applications
} Jorge Francisco Madrigal Diaz, Camille Maurice, Frédéric Lerasle

\section{To cite this version:}

Jorge Francisco Madrigal Diaz, Camille Maurice, Frédéric Lerasle. Hyper-parameter optimization tools comparison for Multiple Object Tracking applications. Machine Vision and Applications, 2019, 30 (2), pp.269-289. 10.1007/s00138-018-0984-1 . hal-01897032

\section{HAL Id: hal-01897032 \\ https://hal.science/hal-01897032}

Submitted on 16 Oct 2018

HAL is a multi-disciplinary open access archive for the deposit and dissemination of scientific research documents, whether they are published or not. The documents may come from teaching and research institutions in France or abroad, or from public or private research centers.
L'archive ouverte pluridisciplinaire $\mathbf{H A L}$, est destinée au dépôt et à la diffusion de documents scientifiques de niveau recherche, publiés ou non, émanant des établissements d'enseignement et de recherche français ou étrangers, des laboratoires publics ou privés. 


\title{
Hyper-parameter optimization tools comparison for Multiple Object Tracking applications
}

\author{
Francisco Madrigal · Camille Maurice - Frederic Lerasle
}

Received: date / Accepted: date

\begin{abstract}
Commonly, when developing an algorithm it is necessary to define a certain number of variables that control its behavior. Optimal parameters result in better performance that could translate into profits for companies, stand out among similar applications or better ranking on algorithm competitions. However, it is not a simple task to find the combination of parameters that provides the best results. Manual tuning could be a stressful and difficult task even for expert users. In this paper we present, evaluate and compare several tools in the literature for hyper-parameter optimization. We focus on 4 tools that have been selected due to their number of citations, code availability and impact on literature: MCMC, SMAC, TPE and Spearmint. We analyze these tools in the context of Multi Object Tracking (MOT) that have not been well studied in the literature. MOT itself has been well-studied topic with multiple parameters to be tuned. We evaluate these tools using public benchmarks such as PETS09 or ETH and using the publicly available source code provided by the authors. We analyze the impact of these tools in terms of stability, performance, and usability, among others. Our results show how the use of these tools change the performance of the application and how this would affect the results of real ranked competitions. Our goal is (1) to encourage the reader to use these tools and (2) to provide a guide that helps to choose the most pertinent tool.
\end{abstract}

This work was carried at LAAS-CNRS and supported by the DGARAPID project SERVAT: Suivi et reconnaissance visuels, adaptatif, temps-réel.

F. Madrigal, C. Maurice, F. Lerasle

LAAS-CNRS

7 av. du Colonel Roche

31400 Toulouse France

E-mail: pacomd@laas.fr
Keywords Stochastic optimization - Parameter tuning · Visual tracking

\section{Introduction}

Multiple Object Tracking (MOT) has several applications that includes surveillance, human-machine interaction, robotics and activity recognition, among others. This is still a hot topic for research due to the existence of many challenges such as occlusion, identity switches or lack precision [41, 25]. MOT community has developed several approaches that challenge some of these issues by proposing different strategies. At the same time, they have created metrics to evaluate the proposal performance, which are known as CLEARMOT [4]. These metrics are relevant because they allow comparing different proposals in a fairly and quantitative way. One particular community named MOTChallenge [22] provides not only the tools to evaluate but also to keep track of the performance of MOT algorithms using the CLEARMOT metrics. Here, the state-of-the-art methods are ranked with respect to their performance. In general, the results are so close that even a small improvement of 2 percent can lead to win or lose 10 positions in the ranking. However, the precision of the results depends on the selection of the meta-parameters in the proposals [26]. Therefore, the ranking could change by selecting only other parameters. Thus, it is difficult to know if a low performance depends on the proposal itself or on an inadequate selection of parameters. Our evaluations show that one parameter configuration tuning can give 50 ID switches while another gives 40, which means a reduction of 20 percent. Commonly the tuning is done manually or, sometimes, the parameters are set based on heuristics or assumptions. Hand tuning is feasible when the number of parameters is small. Otherwise, this could be difficult because of time consumption and requirement of 
expert skills. In either case, there is a possibility of selection bias from the user. Numerical methods are an option to find the best values automatically, performing a furtive search in the (discretized) space of the parameters. However, this requires a high computational cost.

Additionally, the estimation is easy when the proposal is deterministic because it provides the same results every time a sequence is analyzed with the same parameters. However, this is not always the case, for example stochastic approaches, like those based on particle filtering, that give a different result in each evaluation. Therefore, they require analyzing several times the same parameters only to decide if those are good enough. From all of these, the main issues that make the tuning a hard task are: (1) performance impact, (2) computational time of tuning, (3) performance stability, (4) handling of tools and (5) robustness of the tools.

Hyper Parameter Tuning (HPT) could be seen as an optimization process. Here, the goal is to find the set of parameters that maximize (or minimize) a cost function, i.e. the CLEAR-MOT metrics that evaluate a tracking proposal. There are multiple studies on the influence of the (online/ offline) free parameters [3,8,37]. Among all of them, we select the most relevant optimization methods, in terms of number of citations and code availability. Our contribution consists of making an overview (as possible) evaluation of these tools under a MOT context, showing its relevance in this field. MOT approaches could be classified as deterministic or stochastic. The latter is more difficult to tune given its nature of producing a different result in each execution of the same parameters [17]. Therefore, we evaluate HPT tools in this challenging context. Our analysis is based on classic tracking using the Particle Filter (PF). The PF-based proposals commonly involve several parameters [1] and some of them could be correlated. Our study shows the existence of correlation between the parameters and how this affects the search of the optimal parameters. Within the literature, there exist many optimization schemes $[33,6,30]$, which can be roughly classified as local and global optimization [29]. The latter focuses on finding the minimum (or maximum) over the whole parameter space instead of only in the local space. Global optimization [16] could be divided into three groups: Deterministic, Stochastic and Metaheuristic. The first group requires the derivate of the cost function meanwhile the third group is built up based on a heuristic $[40,6]$. In our applicative context, the stochastic nature of PF makes impossible to infer any information of the cost function. Thus, the use of deterministic methods is not suitable and the selection of a heuristic could not work for all the cases. In this paper, our goal is to give an insight of known hyper-parameter optimization tools for multiple object tracking methods. This allows the reader to select the most appropriate tool. Those tools are open source or easy to implement, making them accessible to everyone. We dedicate our study at tracking applications, more precisely at multiple pedestrian tracking, but the results can be applied to other vision modality. We evaluate the performance and provide a statistical analysis of each tool in terms of performance (CLEAR-MOT), stability, robustness and convergence speed among others. To the best of our knowledge, no similar studies have been proposed in the visual tracking community even though optimization tools are essential for fine-tuning [32]. This paper provides a substantial improvement to a previously published conference paper [27]. Here, we evaluate a monocular MOT framework more generic than the multi-camera scheme of [27]. The analysis is broader, with more tools, considering more aspect than the original such as:

- Tool computational time,

- Results repeatability,

- Approach convergence,

- Size of training set,

- Sensitivity to the initial point,

- Evaluation using a combination of metrics and

- Comparison with the state of the art.

The main objective of this work is to provide the reader with an analysis, as detailed as possible, of several optimization tools of hyper parameters. Highlighting the strong features, the weak points to consider and other aspects of interest that can motivate the reader to select the most pertinent tool, mainly in this context of tracking. The analysis focuses on 8 important points that make the tools efficient and practical to use: (1) Convergence speed, (2) Stability, (3) Accuracy, (4) Computational time, (5) Robustness of starting point, (6) training size and (7) number of particles, and (8) Documentation.

The paper is organized as follows: Section 2 gives the details of the selected optimization methods and the description of the corresponding tools. The details of our MOT system and the set of parameters to be optimized are described in section 3. The discussion of the results is presented in section 4. Finally, section 5 describes the conclusion of our analysis.

\section{Hyper-optimization methods}

At first, one can think that the use of an optimization algorithm could be unnecessary, hard to understand or incorporate to each different methods. Our work focuses on analyzing various tools and showing how we can adjust them to the MOT context. There are several strategies for hyperparameter optimization in the literature $[3,8,20]$. We focus on stochastic approaches based on Bayesian optimization. This methodology allows to estimate the global maximum (or minimum) of noisy black-box functions by developing statistical models. It has proven to be a powerful solution in various areas such as machine learning [24,35], robotics [23, 
10], deep learning [13], combinatorial optimization [38, 19], among others. Our goal is to study how its use affects the result of a stochastic MOT framework. The particle filterbased MOT frameworks can be seen as this type of functions because CLEAR-MOT metrics acts as a mapping from tracking results (obtained from the hyper-parameters) to the objective loss function. The stochastic nature of PF could be seen as a noise included in the final output. Stochastic optimization collects information from the observation and builds a model as close as possible to the unknown function [32]. Most of the methods begin with a general model of the function, given a priori. Then, the model is updated according to the observations obtained from the evaluation of the hyper parameters. This process is iterative and the method carefully chooses the next parameters to evaluate. An intelligent algorithm should have a good trade off between the exploration of the hyper-parameter space and exploitation of the cost function, i.e. high values when evaluating the cost function. If this is the case, the algorithm will converge faster, requiring fewer function evaluations and therefore less computational time.

In the literature, there exists several proposals with this goal but only a few provide an open source code. We privilege those approaches with available code. Nevertheless, in the literature there are methods, easy to implement, that could help to find the optimal parameters. To know if a classical method is enough to find reliable parameters, we start our study with a simple stochastic optimization algorithm as a baseline.

\subsection{Bayesian optimization}

At the beginning, it may seem that using a sophisticated optimization algorithm is unnecessary, difficult to understand or use in a particular method. Perhaps a basic method is sufficient to find reliable parameters. To know if this is the case, we begin our study by analyzing a classical stochastic optimization algorithm as a reference.

\subsubsection{Markov Chain Monte Carlo optimization}

Generally, classical methods are model-free, with a large theoretical background [28]. Those are relatively simple and easy to implement. Since we consider our function as a black box, we need a strategy that does not rely neither in the gradient nor heuristics. Thus, we employ stochastic optimization based on Markov Chain Monte Carlo and use this method as a baseline to compare (in terms of performance and easiness of use) against more robust techniques. The Markov Chain Monte Carlo (MCMC) is a set of stochastic methods based on a sampling procedure. It is widely used in the literature, most commonly to determine numerical approximations. In our case, this is the optimal value of the loss function. They do not require prior information of the function, such as the gradient. We can find several MCMC proposals in the literature but we focus on the MetropolisHastings (MH) algorithm [7]. MH approximates a distribution from which direct sampling is difficult or impossible (i.e. the loss function) by drawing samples from a prior known probabilistic distribution. It has a good performance to estimate high dimensional distributions.

Let $f(\lambda)$ be the function that evaluates the hyper parameters $\lambda$. Initially, we choose an arbitrary initial set $\lambda_{0}$ and a probability density $g(\cdot)$ from which we can easily drawn new samples given the previous sample. In our case, we define $g\left(\lambda_{t}\right)$ as a Gaussian distribution centered at the previous $\lambda_{t}$ with a fixed variance. At each iteration, we drawn a candidate sample $\lambda^{*} \sim g\left(\lambda_{t}\right)$ which is used to calculate the acceptance ratio $\alpha=f\left(\lambda^{*}\right) / f\left(\lambda_{t}\right)$. We accept the new candidate $\lambda_{t+1}=\lambda^{*}$ if it improves the function value (i.e. $\alpha>1$ ). If not, we accept it with certain probability given by uniform distribution. The details of the algorithm are available in [7].

\subsubsection{Spearmint}

Previous section describes how MCMC approximates the distribution of the cost functions using sequential samples. Moreover, if we have enough samples, we can assume, following the central limit theorem, that those induce a multivariate Gaussian distribution. This assumption about the distribution of the function is known as the Gaussian process [32]. Some optimization proposals are based on this idea and one in particular is the work of Snoek et al. [35] called Spearmint, which gives and analyzes practical considerations to improve this Bayesian optimization algorithm. Spearmint is well known in the community, having over 1000 citation and additional websites dedicated to explain the tool developed by the author ${ }^{12}$. The objective of Spearmint [35] is to capture the dependence between the hyper parameters $\lambda$ and the cost function $f$. This is achieved with the use of a probability distribution $p(f \mid \lambda)$, which is modeled with the Gaussian process. However, instead of finding the maximum likelihood, the proposal marginalizes the model using slice sampling. This idea allows running many evaluations in parallel each one with a different marginal. This capability of parallelization makes possible to reduce the computational time of some expensive methods such as Deep Learning [36]. Several approaches based on a Gaussian process exploit this mechanism of parallelization [18]. The paper proposes others practical considerations such as the use of a Matern $5 / 2$ kernel instead of squared exponen-

\footnotetext{
1 http://fastml.com/tuning-hyperparams-automatically-withspearmint/

2 http://www.nersc.gov/users/data-analytics/data-analytics-2/deeplearning/hyperparameter-o/spearmint-bayesian/
} 
tial kernel. The later is typically used on Gaussian process regressions but this is unrealistically smooth for several optimization problems. Also, the approach introduces the Expected Improvement (EI) per cost acquisition function that samples not only good parameters but also parameters fast to evaluate.

\subsection{Structure-based Bayesian optimization methods}

Some methods try to describe the manifold of the hyperparameter space through the use of models, generally following an iterative optimization strategy. In each iteration the methods evaluate a hypothesis of the parameters and update the model. After a given number of iterations, these methods propose a final candidate, according to their own methodology, that provides the best performance. This group of methods is called Sequential Model-Based Optimization (SMBO) [19]. These algorithms are recurrently used when the black box cost function is computationally expensive. We evaluate two state-of-the-art methods, which are selected based on their popularity (high number of citations), availability of source code and their good performance.

\section{Sequential Model-based Algorithm Configuration}

One instantiation of SMBO is the Sequential Model-based Algorithm Configuration [19] (SMAC). It is an optimization method based on machine learning with over 600 citations. The tool is under support including the new version 3, which is still in development. This iterative approach considers all the collected information when proposing a new candidate, leading to better parameter estimations. Here, SMAC models the posteriori $p(f \mid \lambda)$ as a Gaussian distribution using random forests (RF) to estimate the empirical mean and variance, one for each tree. This RF-based model improves the performance in discrete optimization, adapts easily to the data and can handle noisy functions with parameters of high dimensionality. SMAC gathers initial data to construct the random forest. Firstly, the set of proposal parameters are sampled from a uniform distribution in a given finite range. scoundly, the samples are divided according to one randomly selected parameter of $\lambda$, which later are used to build the regression trees. This division process is repeated until it reaches a minimum number of samples per branch. The configurations are evaluated using a "expected improvement (EI)" criterion and the most promising are selected. Finally, the best configuration, the one with the highest EI score, is compared against the previous one. The new proposal is accepted if it improves the cost function value. If that is the case, the corresponding tree is used in the next iteration.

\section{Tree-structured Parzen Estimator}

The Tree-structured Parzen Estimator [2] (TPE) is a wellknown model-based method with over 500 citations. Meanwhile SMAC and Spearmint model $p(f \mid \lambda)$ explicitly, i.e. the estimation of the cost function value given the parameters, TPE factors it. Thus, it models the probability of the parameters given that they improve $p\left(\lambda \mid f \geq f^{*}\right)$ or worsen $p\left(\lambda \mid f<f^{*}\right)$ the evaluation of the loss function according to a fixed quantile of the losses observed so far. These two probabilities are modeled with tree-structured Parzen estimators.

In practice, TPE draws, in each iteration, new samples of the parameters and decides which set will try the next iteration. TPE draws samples of $\lambda$ uniformly in the configuration space, therefore it does not require initial guesses or training sets. Then, the samples are evaluated according to cost function $f$. The approach splits the samples in two groups based on their score. The first group has all samples that improve the current score estimation $f^{*}$ meanwhile the second contains the remaining. Then, both groups are used to model the likelihood probability: a model $g(\lambda)=p\left(\lambda \mid f \geq f^{*}\right)$ for the first group with greater values and $l(\lambda) p\left(\lambda \mid f<f^{*}\right)$ for the second group with lower values. The models use a 1-D Parzen estimator to measure the density of the groups through a hierarchical structure. The goal is to create new candidates that are most likely to be in the first group. Thus, new samples of $g(\lambda)$ are drawn at each iteration. The one with the highest improvement is then used in the next iteration. TPE defines the Expected Improvement are the ratio between models, $E I(x)=\frac{l(x)}{g(x)}$.

\subsection{Associated tools}

MCMC is a robust method to estimate the parameter distribution. The implementation is simple and does not require a strong background. Therefore, we create an implementation in $\mathrm{C}++$ using the classic Metropolis-Hastings algorithm [7]. It receives the same input as the others tools such as the limits of the configuration space, number of iterations, initial point and the black box cost function. The configuration space is fixed in the same way as the other tools, where new samples follows a Markov chain, being drawn with a Gaussian distribution with mean in the previous state and a fixed variance. Here, the number of iterations plays an important role in the final results. A small number could lead to an underestimation of the parameter distribution but a large number could generate an over-fitting problem.

Spearmint tool [35] was created by members of the Harvard Intelligent Probabilistic Systems Group. The open source code $^{3}$ requires installing both Python and MongoDB [11].

\footnotetext{
${ }^{3}$ https://github.com/HIPS/Spearmint
} 
The later is a database software that keeps the collected information of Spearmint. MongoDB allows different applications (or threads of the same application) to access the same set of data simultaneously. This allows the communication of many runs of the tools, making parallel optimization possible. Also, it can be used to track the process from the last evaluated iteration, which is useful since it allows to continue the optimization or analyze the results in case there is a problem with the evaluation of the function. Spearmint documentation is very limited, with only a short explanation of how to set up a simple experiment. The included examples give a better idea of the different tool parameters available. It requires defining two files: a parameter file, which set the configuration space limits, and a Python file, which launch the black box function. Additional parameters such as number of iterations are passed as terminal arguments.

SMAC [19] is a publicly available tool for configuration parameter optimization. It has been developed for several years. The stable release SMAC v2 has been published in 2015 and v3 is in development. It is based on Java ${ }^{4}$, making it easy to install and to run on Windows and Unix environment. The authors set documentation such as a quick installation guide, an environment setup and a detailed manual describing the tool, which includes a good description of the output. The framework is easy to use, requiring the definition of a scenario file (in Python) and a parameter file. The first has the information to launch the black box function, the path to the parameter file and optimization options, e.g. the number of iterations. The parameter file describes the configuration space limits, initial point and data type.

TPE [2] is an optimization tool and it forms part of the Hyperopt library ${ }^{5}$ and Optunity library ${ }^{6}$. The later is supported for different languages such as Python, MATLAB, Octave, R, Julia, and Java. However, Optunity is based on Python, which should have been install beforehand. Hyperopt implements TPE algorithm for Python and can be run either serially or in parallel via MongoDB. Both libraries provide simple documentation and small examples of their use. The configuration is simpler than SMAC, limiting it to the call of a minimization function. The function receives as parameters the objective function, number of iterations and the limits of the configuration space.

\section{Study case}

The performance of some frameworks depends on the selection of parameters but finding the optimal configuration could be a hard task even for expert users. Fortunately, the optimal values can be calculated using optimization meth-

\footnotetext{
4 http://www.cs.ubc.ca/labs/beta/Projects/SMAC/v2.10.03/quickstart.h

5 http://hyperopt.github.io/hyperopt/

6 http://optunity.readthedocs.io/
}

ods. There are several proposals in the literature with this goal but only few provide tools (and support) of their methods. In this paper, we evaluate the performance of different hyper-parameter optimization tools. The analysis of the aforementioned tools can be done over any methodology that can be formulated as a cost function. Nevertheless, the relevance of the optimization is more evident with complex methods (such as stochastic) with several parameters. Some Multiple Object Tracking (MOT) methods fit well in this description [21]. MOT is highly studied in the literature because it can be applied in different areas such as security, human-robot interaction, among other. In this context, the goal is to detect and to track pedestrians/people, which could be difficult due to the number of existing issues. To overcome these issues, several methods have been developed and those are usually grouped as deterministic or stochastic [39,34]. The optimal parameters of stochastic methods are more challenging to find because the same set of parameters will give different results in each evaluation. In parallel, the tracking community has created a set of metrics, e.g. CLEAR-MOT [4], to measure the method performance. The metrics allow modeling the tracking system as a cost function. From these highlights, we aim to lead our study in the scope of pedestrians tracking in outdoor environment. Therefore, we have several parameters to optimize with respect to the accuracy measure defined in CLEAR-MOT [4]. The rest of this section gives a description of the tracking system used in our evaluation.

MOTChallenge provides the results of several tracking methods where hundreds of those are based on trackingby-detection. Thus, we follow a decentralized particle filter strategy in the vein of [9]. Therefore, we implement a tracking-by-detection framework where each target state is estimated by a PF in image plane. We define the state as $\mathbf{X}=\{x, y, u, v\}$ where $(x, y)$ are the $2 \mathrm{D}$ image position and $(u, v)$ are the corresponding velocity components. We use the pre-computed detections named ACFINRIA, which are provided by MOTChallenge [22]. The detections are generated with the Aggregate Channel Features pedestrian detector [12], which is trained with the sequences of the INRIA pedestrian training dataset. We always have the same detections for all the experiment, limiting the variability of the algorithm to only the tracker. Thus, the detector is not a vital part of this work because it is not considered in the tunning process.

Each detection is associated to one tracker but only if the distance between them is smaller than a threshold $d H$. We perform the association with the Hungarian algorithm $[9,31,5]$. Fixing a value to $d H$, in the image plane, is not that simple because its value depends on the image size, the frame-rate and how far the camera is from the targets. Moreover, small values can omit correct associations meanwhile high values could lead to a wrong association. So, we 
have to tune $d H$ in such a way that it generalizes the distance for all cases. We create a new tracker for any detection that has been not associated to an existing tracker. We use color information to model the pedestrian appearance with a histogram per target. Then, each tracker contains a reference histogram $H_{r}$, which defines individual appearance, and we build a histogram for any incoming detection $H_{d}$. The observation model of the particle filter measures both histograms using the Bhattacharyya distance. The reference histogram is updated as the weighted sum of both: $H_{r}=$ hur $* H_{r}+\left(1-H_{d}\right)$, where $0<$ hur $<1$ is the histogram updating rate. If the target appearance does not change much over the scene, i.e. constant lighting, we can put hur close to 1 or reducing it otherwise. If the value is too small, we risk adding spurious information in the model, which reduce tracking performance. Finding the optimized value is essential to obtain good results.

The motion model of the PF consists of a random walk model. Therefore, the state is updated as follows: $X_{t}=$ $X_{(t-1)}+\eta_{t}$ where $\eta_{t}=\left\{\eta_{t}^{1}, \eta_{t}^{2}, \eta_{t}^{3}, \eta_{t}^{4}\right\}$ is an independent Gaussian noise for each parameter $\left(\eta_{t}^{*} \sim N\left(0, r w n_{*}\right)\right)$. The random walk noise $r w n_{*}$ allows the tracker to move at different directions with different speed. However, the correct setting depends on the behavior of pedestrians. Small values are good to track slow pedestrians while higher values allow fast targets to be followed. Normally, the sequences have both cases (slow and fast pedestrians) and finding the optimal noise parameters is a hard task.

Our implementation of the particle filter follows the Sequential Importance Resampling scheme, applying a resampling step in each iteration. The number of particles $n p$ plays an important role. In many cases, a large number of particles could improve the state estimation but this also increment the computational cost. Sometimes a small number is adequate for simple scenarios. Therefore, we need to find the number of particles with the best accuracy/computational cost ratio. Finally, this implementation of a basic trackingby-detection framework has 7 parameters to tune: association distance $d H$, histogram updating ratio hur, four random walk noise variables $r w n_{*}$ and the number of particles $n p$. Those components are put together in a vector:

$\lambda=\left\{d H, h u r, r w n_{1}, r w n_{2}, r w n_{3}, r w n_{4}, n p\right\}$,

which is parameter set used in the optimization process. These are the parameters that a MOT system commonly uses but more parameters can be included (i.e. detector threshold) without drastically affecting the performance of the optimization tools.

\section{Evaluation of the tools}

Initially, we describe the data and metrics used to perform the evaluations as well as the methodology. Finally, we present

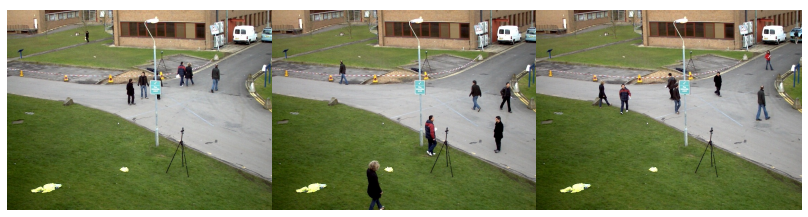

Fig. 1: Examples of sequence S2L1 from PETS 2009 dataset [15]. Left-to-right: views from cameras 1,2,5,6 at frame 49.

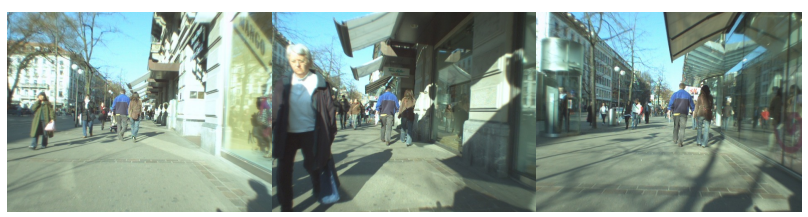

Fig. 2: Examples of sequence Sunny Day from ETH [14].

the results and we discuss them in both quantitative and qualitative aspects.

\subsection{Dataset description}

For all the evaluations of the presented tools, we use two sequences extracted from well-known public datasets: PETS 2009 [15] and ETH [14]. All of these datasets are challenging benchmarks to evaluate the performance of any tracking framework. The PETS2009 dataset has several sequences aimed to a specific goal that goes from single pedestrian tracking to flow estimation. The density of crowdedness varies from sparse to highly dense. We focus on the set S2L1 (see Fig. 1) that consists of 8 synchronized sequences observing a common outdoor scene. Each video has 795 frames recorded at $7 \mathrm{fps}$ with a resolution of $640 \times 480$ pixels. It is a structured scene with three portions of road surrounded by grass. The sequence has a medium density level, with 19 pedestrians, and is oriented to single target tracking with challenging situations, such as clutter occlusions. Among all the sequences, we work with the view 2 . We focus on this sequence because it has been overexploited in the literature. The results have almost reached the ceiling showing small improvement between proposals. Our work is aimed to show how the ranking of the same tracking framework can change with the use of better parameters. The ETH dataset consists of 8 sequences captured by a stereo pair of cameras mounted to a stroller. The camera has a resolution of 640x480 pixels with a frame-rate of 13-14 fps. Each video shows a different place of a busy street. We can see how the camera moves around on the street from a low point of view. These are challenging sequences mainly due to camera position increases the number of total occlusions. We evaluate the tools using the Sunny Day sequence, see Fig. 2. Both 
sequences represent classic scenarios in pedestrian tracking, the first being a static camera located at the distance, with the purpose of surveillance, and the other a mobile camera, with the purpose of interacting with the environment. Therefore, our study analyzes the tools with this kind of videos, which are commonly used in literature.

Calibrated cameras record both datasets. For our framework, we use detections provided by MOTChallenge, which are generated by using the approach of Dollar [12] named Aggregate Channel Features. This pedestrian detector is trained using the INRIA pedestrian training dataset. We perform the evaluation using the kit and ground-truth both provided by MOTChallenge [22].

\subsection{Experimental setup}

We analyze the performance of several parameters optimization tools, where the loss function is as a black box. As mentioned in section 3 , the framework is considered as a black box that takes as input the set of parameters $\lambda$ and returns a measure of the tracking performance. It consists of two parts: (1) a tracking-by-detection system in the same vein as Breitenstein approach [9] and (2) a performance evaluation system, which is carry out using the Multiple Object Tracking Challenge Development Kit provided by MOTChallenge [4]. This section describes the configurations used for each tool and the evaluation metrics used.

\subsubsection{Tool configurations}

All the three tools require to define a search space, either with lower and upper bounds, or covariance. Additionally, SMAC and MCMC require to set initial values, we choose the middle point of the space search. The tools are able to call our tracking function with any test parameters within the specified search space. We need to set a configuration file in order to use any of those optimization tools. In general, it requires to define three aspects: searching space, initial value and type of variable. The searching space must be delimited according to the parameters to evaluate. As mentioned in Eq. 1, we have $\lambda$ with seven variables. From those, only the histogram-update-ratio variable hur is well delimited because it is normalized. For the rest, we can only use our experience to set the limits. Therefore, we define a small configuration space $S$ fixed around from where we expect to find the optimal configuration. In our case, $S$ is set as follows:

$$
\begin{gathered}
S=\{d H \in[0.1,10], \text { hur } \in[0.1,1], \\
\left.r w n_{*} \in[0.1,3], n p \in[10,100]\right\} .
\end{gathered}
$$

However, we do not know if those parameters are the best. Then, we create a larger configuration set $L$ as follows:

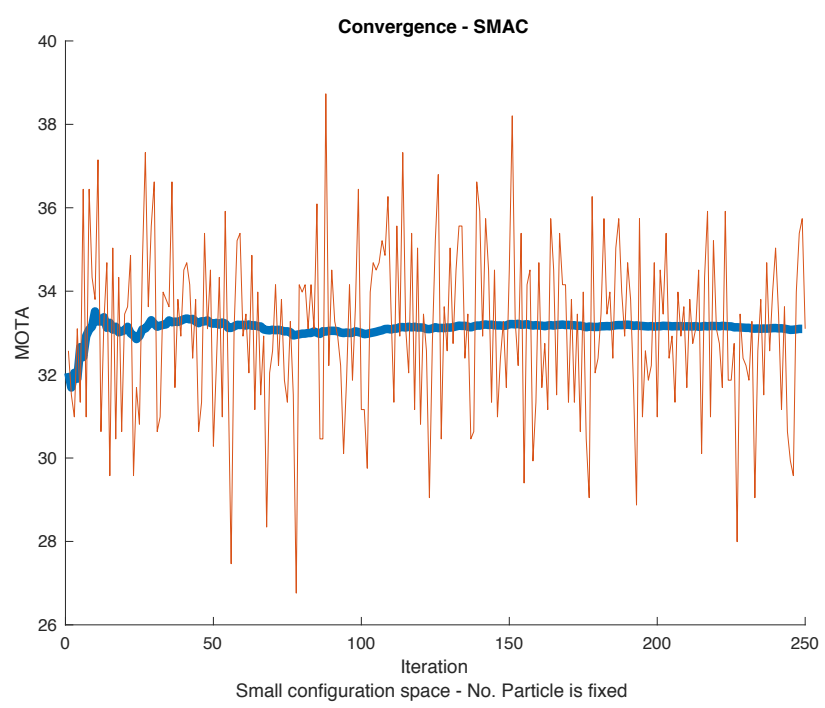

Fig. 3: Evaluation of SMAC using the configuration SF. The function evaluations, using the metric MOTA, are shown in orange. Cumulative distribution is shown in blue.

$$
\begin{gathered}
L=\{d H \in[0.1,20], \text { hur } \in[0.1,1], \\
\left.r w n_{*} \in[0.1,20], \text { np } \in[10,100]\right\} .
\end{gathered}
$$

Also, it could be a correlation between the number of particles $n p$ and the rest of parameters which influences the final results. A higher number could overcome the limits of the other parameters. We analyze this aspect and we label the experiments as $F$ and $N F$ when the number is fixed or not.

\subsubsection{Evaluations and comparison protocol}

In the literature there exists several metrics to measure the performance of tracking approaches. The best-known are the CLEAR-MOT and those are implemented in the MOTChallenge development kit. From all the metrics, we use the Multiple Object Tracking Accuracy (MOTA) metrics as the loss function $f$ because is, commonly, the key metric when comparing several tracking methods. It evaluates the algorithm results with respect to the ground-truth. MOTA combines the information of missed detection, mismatches between detected objects, and false positives. The MOTChallenge [22] ranked by default the results using this metric. When comparing the best positions of the 2D MOT 2015 challenge $^{7}$, we observe that the approaches achieve a similar tracking precision (MOTP). The standard deviation is of 0.9 over 100. Nevertheless, the difference lies in the accuracy that shows a standard deviation of 5.3. By optimizing this metric, the ranking of the methods could change.

\footnotetext{
7 https://motchallenge.net/results/2D_MOT_2015/
} 


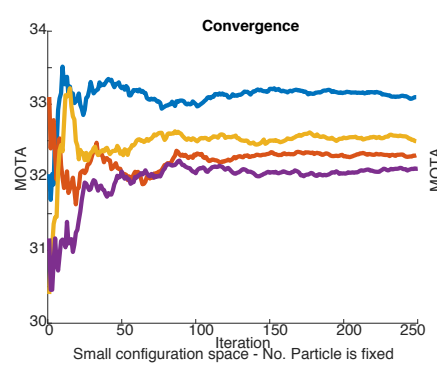

(a) $\mathrm{SF}$

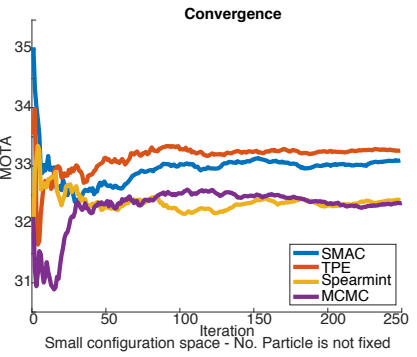

(b) SNF

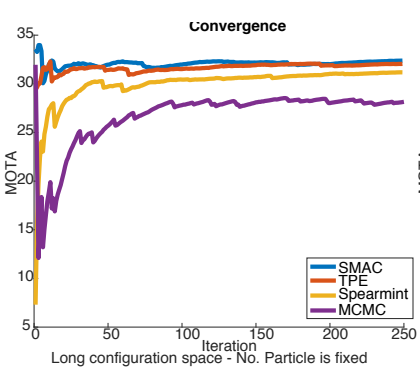

(c) LF

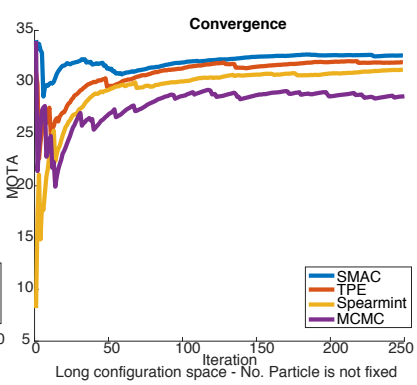

(d) LNF

Fig. 4: Convergence speed evaluation. Cumulative MOTA of SMAC [19], TPE [2], MCMC and Spearmint [35] using PETS09-S2L1 sequence.

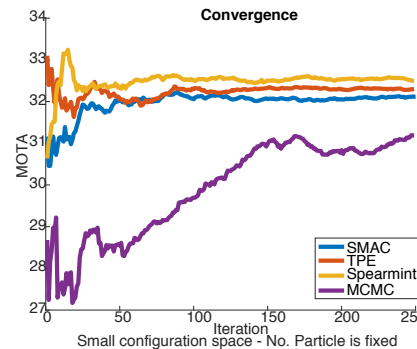

(a) $\mathrm{SF}$

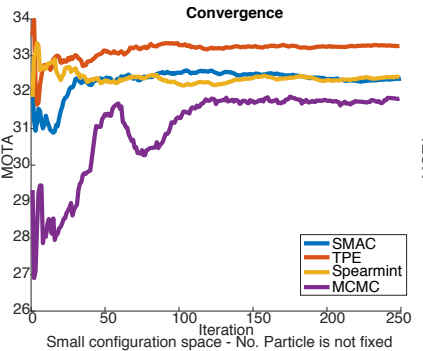

(b) SNF

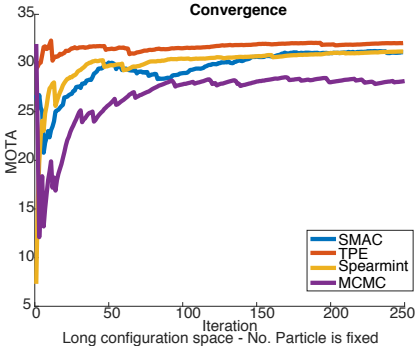

(c) LF

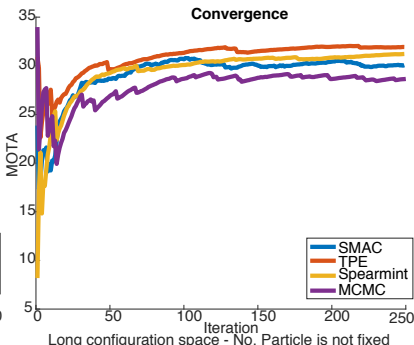

(d) LNF

Fig. 5: Convergence speed evaluation. Cumulative MOTA of SMAC [19], TPE [2], MCMC and Spearmint [35] using ETH Sunny day sequence.

We carry on the evaluations using public sequences. The videos are divided into two sets. One is a training set that is used with the optimization tools to estimate the optimal hyper-parameters. The resulting parameters are then evaluated with the rest of the video that defines the test set. This division allows to avoid over-fitting of the results and boost the configurations that generalize better. Due to the stochastic nature of the method, i.e. tracking framework, we evaluate several times (10) each possible configuration of all the tools. Thus, the results shown are the mean of all the evaluations. This allows to evaluate tools stability and speed of convergence. The later is an important factor that can favor the use of one tool or another. We launch all the evaluations using a Dell Precision Tower 3620, with an Intel Xeon CPU v5 of $3.60 \mathrm{GHz}$ and 8 cores, $16 \mathrm{~GB}$ of RAM over a Linux system (Ubuntu 14.04).

In traditional optimization, the algorithm converges once it reaches a stationary value of the cost function. This is not possible when optimizing stochastic methods. In the literature there are plenty termination criteria but selecting the most appropriate depends on the evaluated functions. Therefore, instead of providing an automatic termination criterion, the tools end after a certain number of iterations, set by the user. SMAC, TPE and Spearmint benefit from additional iterations because that allows them to further explore the configuration space. In theory, the methods will converge to the optimal value when the number of iterations tends to infinity, following the central limit theorem. In order to analyze the convergence of the tools, we use the weak convergence criterion that estimates the cumulative distribution of the measured parameters. We can see an example in Fig. 3 using the results of SMAC. The orange line shows the evaluations of the function (tracking framework), in terms of MOTA, and blue line is the cumulative distribution. We observe that after 50 iterations SMAC starts to stabilize. This means that most of the time SMAC is exploring zones with high MOTA values (33).

\subsection{Results}

We present the results of each hyper-parameter optimization tool using two challenging sequences. On these two views we observe how the tool performs under two different context: static and dynamic cameras. Recall that we analyze the tools in terms of: (1) convergence speed, (2)stability of the optimum value, (3) performance accuracy according to MOTA metric, (4) computational time and influence of (5) starting point, (6) training size and (7) number of particles. Finally, from these evaluations, we propose a ranking of the tools in Tab. 9. 
Table 1: Accuracy. Evaluation over the PETS09 S2L1 sequence.

\begin{tabular}{|c|c|c|c|c|c|c|c|c|c|}
\hline \multirow[b]{2}{*}{ Conf. } & \multirow[b]{2}{*}{ It } & \multicolumn{2}{|c|}{ MCMC } & \multicolumn{2}{|c|}{ SMAC } & \multicolumn{2}{|c|}{ TPE } & \multicolumn{2}{|c|}{ Spearmint } \\
\hline & & Training & Test & Training & Test & Training & Test & Training & Test \\
\hline SF & 250 & 32.75 & 21.65 & 33.45 & 20.70 & 38.20 & 21.12 & 33.47 & 21.94 \\
\hline SF & 500 & 30.28 & 21.39 & 33.55 & 20.58 & 39.44 & 20.58 & 33.53 & 20.30 \\
\hline SF & 1000 & 33.10 & 20.64 & 33.46 & 20.55 & 39.61 & 20.88 & 33.46 & 21.96 \\
\hline SF & 2000 & 33.63 & 21.97 & 33.43 & 20.51 & 39.79 & 20.78 & 34.01 & 21.62 \\
\hline SNF & 250 & 35.04 & 20.95 & 33.97 & 21.31 & 39.44 & 21.67 & 33.66 & 18.78 \\
\hline SNF & 500 & 31.69 & 19.89 & 33.87 & 22.56 & 39.79 & 20.32 & 33.99 & 21.86 \\
\hline SNF & 1000 & 30.99 & 18.72 & 33.80 & 21.76 & 37.68 & 21.45 & 33.96 & 20.53 \\
\hline SNF & 2000 & 34.33 & 22.59 & 33.70 & 21.74 & 40.14 & 20.21 & 33.94 & 22.21 \\
\hline LF & 250 & 34.51 & 21.05 & 33.61 & 21.99 & 36.97 & 22.55 & 33.87 & 22.12 \\
\hline LF & 500 & 33.98 & 19.02 & 33.28 & 22.96 & 38.73 & 21.77 & 34.03 & 22.56 \\
\hline LF & 1000 & 35.56 & 18.82 & 33.56 & 21.75 & 39.09 & 21.62 & 33.91 & 22.28 \\
\hline LF & 2000 & 33.45 & 22.34 & 33.84 & 22.16 & 39.09 & 21.51 & 34.23 & 19.22 \\
\hline LNF & 250 & 32.75 & 21.18 & 33.95 & 20.85 & 36.97 & 21.52 & 34.26 & 5.43 \\
\hline LNF & 500 & 30.46 & 20.79 & 33.92 & 20.55 & 37.32 & 21.40 & 34.27 & 20.76 \\
\hline LNF & 1000 & 33.45 & 20.75 & 33.99 & 21.85 & 38.56 & 21.41 & 34.25 & 22.00 \\
\hline LNF & 2000 & 31.87 & 22.06 & 34.01 & 21.79 & 40.14 & 21.97 & 34.51 & 21.11 \\
\hline \multicolumn{2}{|c|}{ St.D } & 1.53 & 1.17 & 0.23 & 0.75 & 1.05 & 0.60 & 0.30 & 3.98 \\
\hline
\end{tabular}

Table 2: Accuracy. Evaluation over ETH Sunny Day sequence.

\begin{tabular}{|c|c|c|c|c|c|c|c|c|c|}
\hline \multirow{2}{*}{ Conf. } & \multirow{2}{*}{ It } & \multicolumn{2}{|c|}{ MCMC } & \multicolumn{2}{|c|}{ SMAC } & \multicolumn{2}{|c|}{ TPE } & \multicolumn{2}{|c|}{ Spearmint } \\
\hline & & Training & Test & Training & Test & Training & Test & Training & Test \\
\hline SF & 250 & 42.39 & 40.75 & 34.39 & 40.70 & 46.95 & 40.71 & 35.00 & 40.62 \\
\hline SF & 500 & 11.68 & 29.91 & 35.16 & 40.84 & 48.48 & 40.62 & 36.35 & 40.65 \\
\hline SF & 1000 & 41.12 & 40.79 & 35.25 & 40.88 & 46.70 & 40.95 & 35.98 & 40.64 \\
\hline $\mathrm{SF}$ & 2000 & 25.13 & 35.09 & 35.71 & 40.97 & 48.22 & 40.69 & 36.23 & 40.65 \\
\hline SNF & 250 & 43.91 & 40.72 & 34.96 & 40.62 & 45.43 & 41.03 & 36.56 & 40.52 \\
\hline SNF & 500 & 12.69 & 39.83 & 33.73 & 40.77 & 48.22 & 40.66 & 36.34 & 40.81 \\
\hline SNF & 1000 & 29.44 & 40.74 & 34.47 & 40.80 & 47.21 & 40.75 & 37.03 & 40.44 \\
\hline SNF & 2000 & 20.05 & 40.75 & 35.17 & 40.85 & 47.21 & 40.59 & 35.86 & 40.60 \\
\hline LF & 250 & 43.91 & 40.48 & 34.41 & 40.66 & 47.21 & 40.77 & 35.04 & 40.78 \\
\hline LF & 500 & 23.86 & 13.17 & 35.58 & 40.66 & 47.21 & 41.01 & 35.04 & 41.21 \\
\hline LF & 1000 & 27.16 & 40.68 & 35.30 & 40.73 & 47.46 & 40.50 & 37.08 & 40.84 \\
\hline LF & 2000 & 14.98 & 34.04 & 34.66 & 40.81 & 47.62 & 40.67 & 37.97 & 40.58 \\
\hline LNF & 250 & 31.22 & 40.88 & 35.33 & 40.89 & 46.45 & 40.79 & 35.92 & 41.02 \\
\hline LNF & 500 & 14.72 & 36.34 & 33.75 & 40.72 & 49.49 & 40.82 & 36.05 & 40.23 \\
\hline LNF & 1000 & 31.73 & 40.66 & 35.22 & 40.75 & 47.46 & 40.80 & 36.38 & 40.57 \\
\hline LNF & 2000 & 22.59 & 40.22 & 35.90 & 40.89 & 48.73 & 40.75 & 37.73 & 36.30 \\
\hline \multicolumn{2}{|c|}{ St. D. } & 10.81 & 6.95 & 0.62 & 0.10 & 0.94 & 0.14 & 0.84 & 1.08 \\
\hline
\end{tabular}

Convergence speed. We analyze the weak convergence criterion for all the tools under different configurations $S$ and $L$ with the number of particle fixed $F$ and non-fixed $N F$. Figs. 4 and 5 shows the results. We observe that SMAC and TPE stabilize faster and to the highest values most of the time. Meanwhile, MCMC stabilizes slower than the others and Spearmint shows an average performance in most of the cases.

Stability. In Fig. 6 and 7 we analyze the performance stability of the tools for the four configurations $S F, S N F, L F$, $L N F$. The evaluations are done with 1000 iterations, meaning that each tool evaluate 1000 times the cost function. The MOTA output is used to build up a box plot that represents MOTA distribution. Both Figs. show the median (red line), the first and third quantiles (blue box) and the outliers (red crosses). We can observe that all the boxes of MCMC are the bigger than the others.

Accuracy. The previous results indicate that TPE and SMAC have a better convergence rate with an efficient use of each iteration. We can observe in Tables 1 and 2 the results in terms of precision using the MOTA metric. We evaluate the tools for all the configurations and a fixed number of iterations using the training set (i.e. the first part of the sequence). At the end, the tool gives a configuration and its associated MOTA value. We evaluate 10 times each tool and we show the mean value in the training column. This number offers a good compromise between the computation time and the number of evaluations needed to estimate the behavior of 


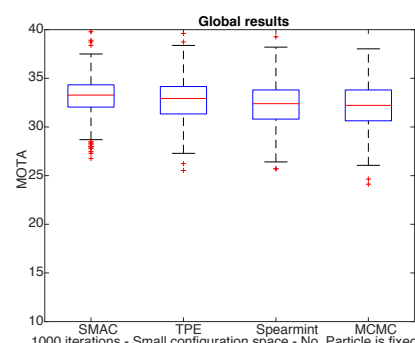

(a) $\mathrm{SF}$

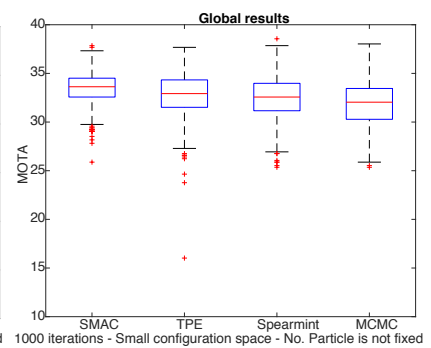

(b) SNF

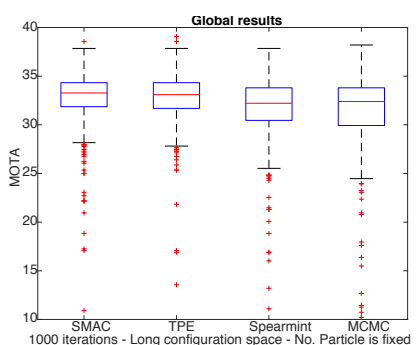

(c) $\mathrm{LF}$

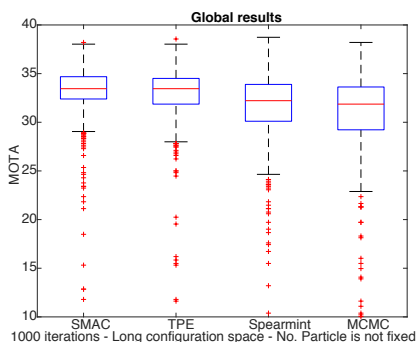

(d) LNF

Fig. 6: Performance stability. Analysis using PETS sequence. We show the distribution of the MOTA values obtained with 1000 iteration of each tool: SMAC [19], TPE [2], MCMC and Spearmint [35].

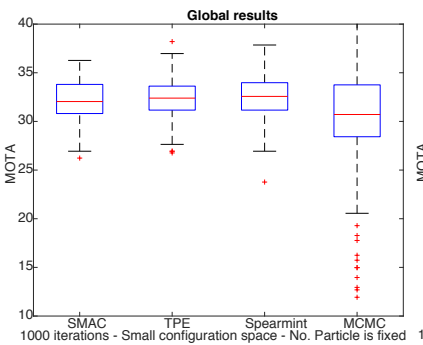

(a) $\mathrm{SF}$

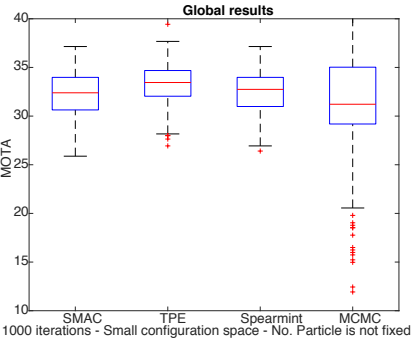

(b) SNF

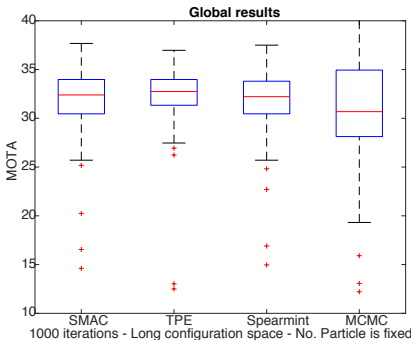

(c) LF

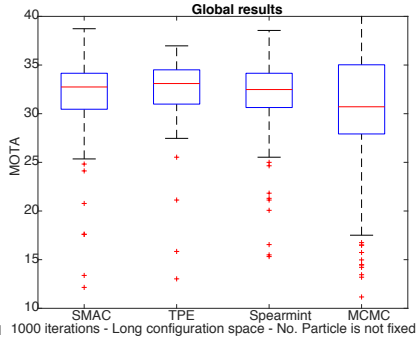

(d) LNF

Fig. 7: Performance stability. Analysis using ETH Sunny day sequence. We show the distribution of the MOTA values obtained with 1000 iteration of each tool: SMAC [19], TPE [2], MCMC and Spearmint [35].

these stochastic tools. In all the cases, the variance is less than 0.2 . Then, we evaluate 30 times the parameters given by the tools using the test set. The results are shown in the test column. Last row shows the mean standard deviation of each column.

We analyze the whole sequences using the best parameters of each tools. The results are shown in Tables 3 and 4. In both cases, SMAC and TPE show the best results. The results displayed at the webpage of MOTChallenge are the average values across all sequences. Nevertheless, they provide the individual results of each single video. We calculate the mean and standard deviation of all provided results for the ETH and PETS sequences. The results are shown in the last two lines of the tables.

Computational time. The Tab. 5 and Fig. 8 show the mean computational time used for each configuration $(S, L)$ using the PETS sequence. The time of each iteration of MCMC is practically the same, having a linear increment with respect to the number of iterations. Meanwhile SMAC is a bit slower than MCMC, but it is faster than the rest of the tools. TPE has a good performance in comparison. However, Spearmint computational time increases at each iteration. This could be a limitation. In our experiment we observe that Spearmint performs, in general, better with more iterations. The results with ETH sequence are similar and the only difference is, therefore, a different number of frames to process.
Influence of starting point. In optimization, the initial parameters could influence the final results. TPE and Spearmint use the lower limits of $S$ and $L$ as starting point by default and this cannot be changed. Nevertheless, SMAC and MCMC allow to set the initial point. We evaluate 10 parameters randomly generated 5 times each using the training set with 500 iterations. We use the large configuration without fixing the number of particles because this setting has shown the best performance. The tool results are evaluated 30 times using the test set. We calculate the mean and standard deviation of the MOTA metric of the training and test set. The results are shown in Table 6 for PETS and ETH sequences respectively.

Influence of the training size. The tool performance increases when more data is available. However, this is not possible in all cases. We analyze how training size impacts the results. We evaluate 4 sizes corresponding to the initial 5, 10, 15 and 20 percent of the video. The Table 7 shows the results for both sequences. We observe that all the tools work well after 10 percent. MCMC, SMAC and TPE increase the precision when more frames are used.

Influence of the number of particles. In our evaluations, we fixed the number of particles to 30 . This is motivated because this number gives a good trade-off between computational time and precision. However, a variation on the used 
Table 3: Accuracy. Evaluation over the whole PETS09-S2L1 sequence.

\begin{tabular}{llrllllllrrrr}
\multicolumn{1}{c}{ Method } & Conf. & IDF1 & Rcll & Prcn & MT & ML & FP & FN & IDs & MOTA & MOTP & MOTAL \\
\hline MCMC & SF & 43.22 & 75.07 & 65.12 & 7.27 & 0.43 & 1869.40 & 1158.60 & 39.97 & $\mathbf{3 3 . 9 8}$ & 69.37 & $\mathbf{3 4 . 8 0}$ \\
MCMC & SNF & 42.77 & 74.49 & 65.18 & 7.53 & 1.57 & 1849.30 & 1185.03 & 40.53 & 33.83 & 69.55 & 34.67 \\
MCMC & LN & 42.33 & 74.15 & 65.03 & 7.17 & 1.57 & 1853.27 & 1201.23 & 40.73 & 33.39 & 69.37 & 34.24 \\
MCMC & LNF & 42.38 & 74.29 & 65.24 & 7.07 & 1.83 & 1838.97 & 1194.60 & 40.57 & 33.85 & 69.47 & 34.68 \\
SMAC & SF & 42.41 & 74.88 & 64.78 & 7.57 & 0.67 & 1892.13 & 1167.50 & 44.13 & 33.21 & 69.39 & 34.12 \\
SMAC & SNF & 43.15 & 75.33 & 65.24 & 7.43 & 0.60 & 1865.17 & 1146.03 & 43.53 & 34.26 & 69.56 & 35.17 \\
SMAC & LN & 43.82 & 75.11 & 65.12 & 7.57 & 0.53 & 1868.90 & 1157.03 & 40.90 & 34.01 & 69.39 & 34.84 \\
SMAC & LNF & 43.97 & 75.80 & 65.60 & 7.60 & 0.20 & 1847.27 & 1125.07 & 41.93 & $\mathbf{3 5 . 1 3}$ & 69.61 & $\mathbf{3 6 . 0 0}$ \\
TPE & SF & 43.06 & 75.01 & 65.01 & 7.53 & 0.53 & 1876.47 & 1160.63 & 44.67 & 33.68 & 69.35 & 34.61 \\
TPE & SNF & 43.14 & 75.10 & 64.88 & 7.70 & 1.37 & 1888.47 & 1157.40 & 46.83 & 33.44 & 69.52 & 34.42 \\
TPE & LN & 44.05 & 75.09 & 65.15 & 7.57 & 0.53 & 1866.33 & 1157.53 & 41.30 & 34.05 & 69.33 & 34.89 \\
TPE & LNF & 43.69 & 75.49 & 65.44 & 7.33 & 0.43 & 1852.50 & 1139.30 & 42.27 & $\mathbf{3 4 . 7 0}$ & 69.57 & $\mathbf{3 5 . 5 8}$ \\
Spearmint & SF & 41.27 & 74.76 & 64.62 & 7.50 & 1.43 & 1901.47 & 1173.47 & 50.93 & 32.73 & 69.37 & 33.79 \\
Spearmint & SNF & 42.45 & 75.33 & 65.21 & 7.53 & 0.50 & 1867.40 & 1146.23 & 43.83 & 34.21 & 69.61 & 35.12 \\
Spearmint & LN & 43.36 & 74.90 & 64.91 & 7.53 & 0.83 & 1881.83 & 1165.90 & 42.40 & 33.50 & 69.41 & 34.37 \\
Spearmint & LNF & 42.75 & 75.73 & 65.33 & 7.54 & 0.53 & 1857.40 & 1141.13 & 43.13 & $\mathbf{3 4 . 3 3}$ & 69.61 & $\mathbf{3 5 . 1 7}$ \\
\hline MOTChallenge & Mean & - & 83.29 & 81.24 & 14 & - & 1029.17 & 775 & 167 & 57.13 & 71.17 & 60.701 \\
MOTChallenge & St. D. & - & 5.541 & 10.77 & 2.08 & - & 894.224 & 261.054 & 108.5 & 16.23 & 0.442 & 15.376
\end{tabular}

Table 4: Accuracy. Evaluation over the whole ETH Sunny day sequence.

\begin{tabular}{|c|c|c|c|c|c|c|c|c|c|c|c|c|}
\hline Method & Conf. & IDF1 & Rcll & Prcn & MT & ML & FP & FN & IDs & MOTA & MOTP & MOTAL \\
\hline MCMC & SF & 53.02 & 71.67 & 85.95 & 9.10 & 9 & 217.40 & 526.50 & 11.10 & 59.37 & 78.19 & 59.90 \\
\hline MCMC & SNF & 54 & 71.16 & 86.3 & 10.5 & 8.8 & 209.9 & 535.9 & 10.2 & 59.31 & 78.19 & 59.8 \\
\hline MCMC & $\mathrm{LN}$ & 52.74 & 71.82 & 86.03 & 9.2 & 8.9 & 217.2 & 523.7 & 11.3 & 59.51 & 78.06 & 60.07 \\
\hline MCMC & LNF & 52.15 & 71.28 & 86.1 & 9 & 9 & 213.8 & 533.5 & 10.6 & 59.21 & 77.93 & 59.71 \\
\hline SMAC & $\mathrm{SF}$ & 52.78 & 71.76 & 86.14 & 9 & 9 & 214.5 & 524.7 & 10.4 & 59.64 & 77.97 & 60.16 \\
\hline SMAC & SNF & 52.47 & 71.72 & 86.03 & 9.4 & 9 & 216.4 & 525.4 & 11.5 & 59.46 & 78.2 & 60.02 \\
\hline SMAC & LN & 53.2 & 71.46 & 86.27 & 8.9 & 9 & 211.2 & 530.4 & 10.5 & 59.52 & 77.9 & 60.01 \\
\hline SMAC & LNF & 52.89 & 71.72 & 86.01 & 9.10 & 8.9 & 216.90 & 525.80 & 11.60 & 59.40 & 78.06 & 59.96 \\
\hline TPE & $\mathrm{SF}$ & 52.73 & 71.74 & 86.16 & 9.1 & 9 & 214.5 & 524.9 & 10.9 & 59.6 & 78.07 & 60.16 \\
\hline TPE & SNF & 52.92 & 71.77 & 86.25 & 9.2 & 8.9 & 212.4 & 524.6 & 11.5 & 59.72 & 78.09 & 60.27 \\
\hline TPE & LN & 52.89 & 71.6 & 86.11 & 9 & 9 & 214.7 & 527.9 & 11.4 & 59.42 & 78.07 & 59.97 \\
\hline TPE & LNF & 53.02 & 71.78 & 86.2 & 8.8 & 9 & 213.6 & 524.2 & 11.2 & 59.69 & 77.92 & 60.24 \\
\hline Spearmint & $\mathrm{SF}$ & 52.69 & 71.62 & 85.79 & 9 & 9 & 220.6 & 527.2 & 10.8 & 59.17 & 78.13 & 59.7 \\
\hline Spearmint & SNF & 52.87 & 71.65 & 86.04 & 9 & 9 & 216.2 & 526.9 & 11 & 59.41 & 78.07 & 59.95 \\
\hline Spearmint & $\mathrm{LN}$ & 52.3 & 71.77 & 86.25 & 9.1 & 9 & 212.8 & 524.5 & 11.2 & 59.31 & 78.25 & 59.26 \\
\hline Spearmint & LNF & 52.65 & 71.85 & 86.48 & 9.2 & 9 & 218.7 & 523.1 & 11.2 & 58.98 & 78.41 & 59.55 \\
\hline MOTChallenge & Mean & - & 61.13 & 86.26 & 5.33 & 8.17 & 211.67 & 736.33 & 41.50 & 47.66 & 77.77 & 49.78 \\
\hline MOTChallenge & St. D. & - & 3.22 & 10.40 & 2.21 & 1.34 & 211.58 & 63.17 & 40.96 & 10.87 & 1.88 & 10.64 \\
\hline
\end{tabular}

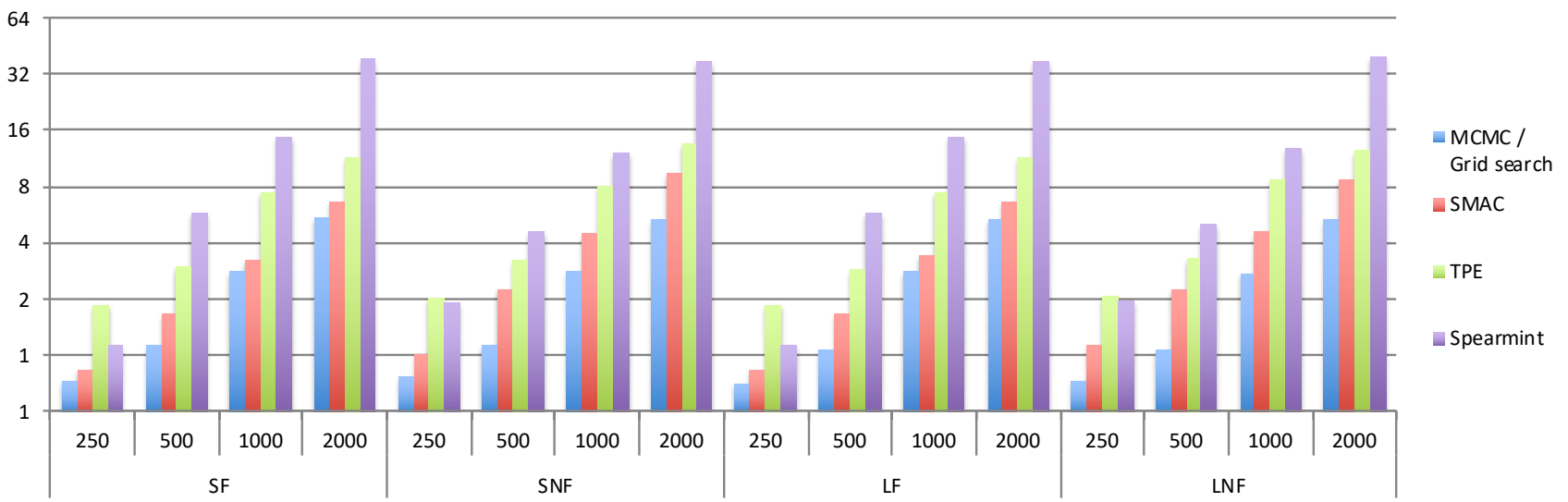

Fig. 8: Computational time, in hours, used by the tools with the PETS09 S2-L1 sequence 
value changes the performance of the results. We analyze this behavior testing different number of particles under the large configuration. We focus into analysis of SMAC and TPE because these have shown the best performance until now. The optimized hyper-parameters are then evaluated over the whole sequence of PETS. The mean of 30 evaluations is shown in the Table 8.

Table 5: Computational time, in hours, used by the tools with the PETS09 S2-L1 sequence.

\begin{tabular}{|rrrrrr} 
& & \multicolumn{4}{c}{ Time (hours) } \\
Conf. & No. Iter. & MCMC & SMAC & TPE & Spearmint \\
& 250 & 0.73 & 0.83 & 1.86 & 1.14 \\
SF & 500 & 1.12 & 1.69 & 2.96 & 5.83 \\
& 1000 & 2.79 & 3.27 & 7.34 & 14.78 \\
& 2000 & 5.41 & $\mathbf{6 . 7 2}$ & $\mathbf{1 1 . 6 0}$ & 38.36 \\
& 250 & 0.77 & 1.02 & 2.02 & 1.92 \\
& 500 & 1.14 & 2.26 & 3.21 & 4.64 \\
SNF & 1000 & 2.86 & 4.46 & 8.00 & 12.24 \\
& 2000 & 5.37 & $\mathbf{9 . 4 2}$ & $\mathbf{1 3 . 3 8}$ & 37.17 \\
& 250 & 0.70 & 0.83 & 1.85 & 1.15 \\
LF & 500 & 1.09 & 1.69 & 2.92 & 5.80 \\
& 1000 & 2.83 & 3.40 & 7.31 & 14.71 \\
& 2000 & 5.33 & $\mathbf{6 . 6 3}$ & $\mathbf{1 1 . 5 2}$ & 37.17 \\
& 250 & 0.72 & 1.15 & 2.08 & 1.98 \\
\hline \multirow{4}{*}{ LNF } & 500 & 1.08 & 2.26 & 3.31 & 4.99 \\
& 1000 & 2.77 & 4.60 & 8.66 & 12.78 \\
& 2000 & 5.24 & $\mathbf{8 . 7 3}$ & $\mathbf{1 2 . 5 7}$ & 39.95
\end{tabular}

Table 6: Influence of starting point. Mean MOTA results with 10 parameter sets randomly generated. We test them with the large configuration with not fixed particles and 500 iterations.

\begin{tabular}{lccccc} 
& & \multicolumn{2}{c}{ Training } & \multicolumn{2}{c}{ Test } \\
Sequence & Method & MOTA & St. D. & MOTA & St. D. \\
\hline \hline PETS & MCMC & 33.95 & 0.72 & 19.13 & 1.56 \\
PETS & SMAC & 34.05 & 0.08 & 22.39 & 0.66 \\
\hline ETH & MCMC & 19.77 & 6.37 & 27.92 & 14.71 \\
ETH & SMAC & 34.81 & 0.76 & 40.23 & 0.30
\end{tabular}

\subsection{Discussion}

Hyper-parameters optimization tools are designed to support proposals that require tuning multiple variables. Therefore, they must be intuitive to use and adapt well to different scenarios. Our proposal gives an original insight of relevant tools for MOT frameworks. This section highlights some interesting aspect of the tools based on the previous results.

Convergence speed. The analysis of the weak convergence criteria (see Figs. 4 and 5) show interesting results. Both
SMAC and TPE find the highest values of MOTA most of the time. They converge faster than the rest on any configuration. Our MCMC baseline takes longer to stabilize and the cumulative distribution reaches lower MOTA values compared to the others. Spearmint can find in the short configuration high MOTA values in few iterations. However, it takes more time to process the large one. Furthermore, the small configuration is selected around where it is expected to have the optimal parameter configuration. It is set based on expert knowledge about the parameters of the MOT framework. This prior information helps optimization tools to converge faster with more stable MOTA values compared to the larger configuration. However, if these limits cannot be clearly delineated or if we know little about the effect of the parameters on the system, we can use a larger configuration and get the same results with just more iterations. The number of particles plays an important factor. The initial intuition to fix it is to reduce the number of parameters to optimize. However, we observe that the results labeled as NF (Non-Fixed) converge more rapidly than the others. This is because the tools use this variable to compensate the rest. More clearly, a high number of particles are considered when the other parameters do not perform well in the tracking system. For example, the tools test with more particles when the noise of the random walk parameters is small. Moreover, fixing or not fixing this parameter changes the behavior of TPE by improving its performances in the short configuration for both sequences. Thus, we conclude that both TPE and SMAC generally converge to the optimal MOTA value in a faster way than the other approaches. Therefore, these tools are recommended when a result with few function evaluations is required.

Stability. Ideally, tools should explore the configuration space efficiently, evaluating promising parameters. If this is the case, we expect that the distribution of the evaluations follows a Gaussian distribution with a small variance. Otherwise, the variance increases when evaluating suboptimal parameters, which translates in a waste of iterations. The box plot quantifies this analysis in the Figs. 6-7. This shows how MCMC is, overall, unstable to achieve a proper solution and constantly evaluating, and accepting, hyperparameters with low MOTA values. On the other hand, TPE is more robust, using wisely each iteration to explore promising parameters. SMAC outperforms the rest with respect to PETS dataset, showing a smaller variance than the others. It finds stable parameters that generalize well in this scenario with static camera. However, SMAC has problems to handle the moving camera of ETH sequence. This is because it tries to optimize as quickly as possible the variables related to distance. This makes it oscillate between configurations adapted for moments where the targets are far from the camera and configurations for targets close to the camera. Thus, it is advis- 
Table 7: Influence of the training size. Performance evaluation changing the size of training set.

\begin{tabular}{|c|c|c|c|c|c|c|c|c|c|}
\hline \multirow{2}{*}{ Seq. } & \multirow{2}{*}{$\%$} & \multicolumn{2}{|c|}{ MCMC } & \multicolumn{2}{|c|}{ SMAC } & \multicolumn{2}{|c|}{ TPE } & \multicolumn{2}{|c|}{ Spearmint } \\
\hline & & Training & Test & Training & Test & Training & Test & Training & Test \\
\hline PETS & 5 & 30.97 & 17.58 & 30.69 & 21.35 & 37.85 & 20.10 & 7.09 & 18.58 \\
\hline PETS & 10 & 31.13 & 17.60 & 31.18 & 21.88 & 39.09 & 19.97 & 34.30 & 21.21 \\
\hline PETS & 15 & 31.53 & 18.93 & 32.46 & 22.09 & 37.15 & 22.60 & 34.85 & 22.56 \\
\hline PETS & 20 & 31.89 & 19.47 & 33.80 & 22.19 & 38.20 & 22.76 & 32.11 & 20.87 \\
\hline ETH & 5 & 6.80 & 18.20 & 17.88 & 28.09 & 27.42 & 18.2 & 33.13 & 38.45 \\
\hline ETH & 10 & 21.22 & 19.20 & 34.87 & 38.26 & 48.58 & 19.2 & 33.11 & 40.08 \\
\hline ETH & 15 & 23.53 & 38.95 & 35.42 & 40.17 & 46.36 & 38.95 & 33.07 & 39.32 \\
\hline ETH & 20 & 37.22 & 39.71 & 39.28 & 40.53 & 40.17 & 39.19 & 32.82 & 39.94 \\
\hline
\end{tabular}

able to use SMAC or TPE for methods whose cost function contains noise, i.e. stochastic methods and TPE is more reliable with non-static sequences.

Accuracy. From Tabs. 1-2, we observe that the results of MCMC are unstable, arriving to different values at each iteration. This is due to its implementation does not consider noisy function evaluations. MCMC results are unstable, reaching different values in each iteration. This is because their implementation does not consider that evaluations of functions have noise, i.e., two evaluations of the same parameters result in two different MOTA values. Then, its performance is affected by the stochastic output of the particle filter-based tracker. One way to overcome this issue is to approximate the distribution of the parameter set using, for example, MCMC. This will require a larger number of evaluations, which makes it computationally expensive. Also, MCMC training results show that it needs a few iterations to converge at any configuration (small or large and fixed or non-fixed). A greater number of iterations generates overfitting, reducing its performance. Spearmint provides more stable results, with a standard deviation of 0.3 . However, the given parameters do not generalize well the rest of the sequence. Moreover, in this case the number of iterations needed to obtain good results is proportional to the complexity of the configuration, i.e., the large non-fixed configuration needs more iterations to reach the optimal value. Unlike, TPE converges slowly but it provides robust parameters. It presents the highest values on the training set but this is because TPE reports the highest value found. Meanwhile, SMAC and Spearmint report the expected mean value. SMAC appears to be more stable than the others in both training and testing sets. The results from the training set are generally consistent with those from the testing set. From Tables 3 and 4, we can compare our results with those provided by MOTChallenge. The obtained MOTA in PETS sequence is in the lower middle with respect to the others, meanwhile, out tracking method is in the upper middle with the ETH sequence. This is due to the fact that the sequence of PETS has been evaluated by dozens of methods, each one contributing a slight increase with respect to the previous one. However, ETH sequence has been analyzed with only few methods, which are not at the top of the ranking. Our system does not attempt to compete with the methods of literature, but to show how the same framework can outperform itself by using better parameters. From Tab. 3 we observe a difference of more than 2 percent between the worst and best result. It may not seem to be much, but if we check the MOTChallenge ranking, scores between approaches are only different by a few decimals. Therefore, this slight change over several sequences could end in an increase of 5 positions in this ranking. In general, all methods give a good performance, but among them SMAC gives a more accurate result. Therefore, we recommend its use when the main goal is the accuracy.

We analyze the performance of the tools considering other metrics and making a combination between them. However, the MOTA metric includes many features that range from false positives, false negatives and identity switch. Those are strongly related to other metrics and therefore the results obtained reached the same conclusions as when using MOTA exclusively.

Computational time. The time used for each tool plays an important role when selecting which one to use. When comparing the configurations $F$ and $N F$ of Fig. 8, we observe MCMC and Spearmint have a similar cost. However, SMAC and TPE present a different behavior. The CPU time is larger when the number is not fixed, the Table 5 highlights this phenomenon. This is because both tools need to build a model for 7 parameters instead of 6 . Thus, the computational time of both tools will increase according to the number of parameters that will be optimized. In this case, SMAC is the one with the largest increment.

Influence of starting point. The choice of starting point influences the outcome of most optimization systems. A good method should be able to handle this and converge to the global optimum, avoiding getting stuck in the local minimum. When comparing Table 6 against Tables 1 and 2, we observe that SMAC and MCMC show a similar behavior regardless the initial parameters. Moreover, the analysis of 
Table 8: Influence of the number of particles. Evaluation on PETS09 sequence fixing the number of particles.

\begin{tabular}{|c|c|c|c|c|c|c|c|c|c|c|c|c|c|c|}
\hline Method & Conf. & No. Part. & Time (hrs.) & IDF1 & Rcll & Pren & MT & ML & FP & FN & IDs & MOTA & MOTP & MOTAL \\
\hline TPE & $\mathrm{LF}$ & 10 & 1.94 & 42.68 & 73.99 & 64.12 & 7.20 & 0.90 & 1923.9 & 1209 & 44 & 31.64 & 69.09 & 32.55 \\
\hline TPE & $\mathrm{LF}$ & 30 & 2.17 & 44.05 & 75.09 & 65.15 & 7.57 & 0.53 & 1866.3 & 1158 & 41.30 & 34.05 & 69.33 & 34.9 \\
\hline TPE & $\mathrm{LF}$ & 50 & 2.38 & 43.91 & 75.49 & 65.51 & 7.50 & 0.40 & 1847.1 & 1139 & 40.3 & 34.88 & 69.40 & 35.7 \\
\hline TPE & $\mathrm{LF}$ & 70 & 2.69 & 42.09 & 74.92 & 65.01 & 7.60 & 1.20 & 1873.5 & 1165 & 45 & 34.63 & 69.57 & 35.56 \\
\hline TPE & $\mathrm{LF}$ & 100 & 3.17 & 42.55 & 75.69 & 65.39 & 7.30 & 0.10 & 1861 & 1130 & 41.6 & 34.74 & 69.66 & 35.62 \\
\hline SMAC & LF & 10 & 1.38 & 44.33 & 74.38 & 64.74 & 6.90 & 0.80 & 1883.9 & 1190 & 38.7 & 33.02 & 69.10 & 33.82 \\
\hline SMAC & $\mathrm{LF}$ & 30 & 1.69 & 43.82 & 75.11 & 65.12 & 7.57 & 0.53 & 1868.9 & 1157 & 40.9 & 34.01 & 69.39 & 34.84 \\
\hline SMAC & $\mathrm{LF}$ & 50 & 1.82 & 42.47 & 75.46 & 65.36 & 8.00 & 1.00 & 1857.7 & 1141 & 44.1 & 34.54 & 69.54 & 35.44 \\
\hline SMAC & $\mathrm{LF}$ & 70 & 2.56 & 45.02 & 75.62 & 65.46 & 7.20 & 0.40 & 1854.7 & 1132 & 40.5 & 34.86 & 69.57 & 35.71 \\
\hline SMAC & $\mathrm{LF}$ & 100 & 2.66 & 43.52 & 75.82 & 65.46 & 7.80 & 0.60 & 1859.4 & 1124 & 43.9 & 34.87 & 69.55 & 35.77 \\
\hline
\end{tabular}

\begin{tabular}{|l|c|c|c|c|c|c|c|c|}
\hline Tool & Convergence & Stability & Accuracy & CPU cost & Init. Cond. & Training size & Accessibility & Rank \\
\hline \hline MCMC [7] & + & + & + & +++ & + & + & + & 4 \\
\hline Spearmint [35] & ++ & ++ & ++ & + & - & ++ & + & 3 \\
\hline SMAC [19] & +++ & ++ & +++ & +++ & +++ & +++ & +++ & 1 \\
\hline TPE [2] & +++ & +++ & ++ & ++ & - & +++ & ++ & 2 \\
\hline
\end{tabular}

Table 9: Evaluation summary: (+) Good, (++) Better, (+++) Best.

SMAC in terms of convergence shows that the only difference is that the number of iterations varies with respect to how far it is from the optimal minimum. However, MCMC still has problems to converge, staying on local minimums for several iterations and over-fitting problems.

Influence of training size. It is natural that increasing the size of training data also increases the accuracy. Our results show this same effect. Also, we can see that starting from 10 percent, the increase is small. This is because the initial 10 percent of the evaluated sequences describes, in a general way, the movement of pedestrians in the scene. And likewise the optimal parameters that should be used.

Influence of the number of particles. The number of particles plays an important role in this type of trackers. When fixing this number, we observe how the results improve when more particles are used. However, this also increases the computational time. Furthermore, analyzing the parameter sets evaluated by the tools without fixing this number, we noticed that many good candidates maintained a low number of particles, in the range of 10 to 50 .

Accessibility. Overall, the tools define the search space but only SMAC and (our implementation of) MCMC can set the initial parameters. In contrast, TPE and Spearmint use the lower limit as initial position making them simpler to configure. SMAC has the best ratio between CPU time and performance, followed by TPE. On the contrary, Spearmint has a constant increase in time in each iteration. This is a problem because its results are better with more iterations. TPE has the advantage that it can be call as a function. In comparison, SMAC and Spearmint are more complex, requiring to create a set of files and folders with specific formats. The documentation is a vital part when we decide to use a new tool. In this aspect, SMAC surpasses the rest with detailed official documentation, active discussion forums and developer support. MCMC has a strong background in the literature with many examples for different topics. TPE has a small but good documentation of its use. However, Spearmint's official documentation is almost non-existent, mostly limited to the installation and how to launch a simple example. Additionally, SMAC and Spearmint collect information from each iteration, which is available so that the user can observe and analyze it. SMAC is more organized, separating specific data in many easy-to-understand files. Meanwhile, Spearmint provides summary files of each iteration and a database with the global information. TPE and MCMC do not provide information as exhaustive as the other two, but simple results such as the parameters and the value of the cost function in each iteration. From the above, we created Tab. 9 that summarizes our experience when using these tools. We consider that SMAC gives the best compromise with respect to our criteria mentioned on Tab. 9. It is the easiest to use despite the number of files to configure. The documentation is extensive and detailed and it has an active community supporting it. In addition, SMAC shows to be more stable in both convergence efficiency and exploration, which is important for the repeatability of the results. We consider TPE as a good option but we rank it in second place. It offers a wide range of possibilities but the limited documentation makes it difficult to use for different scenarios. It also has a good convergence rate, even surpassing SMAC in some cases. Spearmint gives good performance but, according to our results, not as SMAC and TPE. Furthermore, the small documentation and the computational time are characteris- 
tics that make it difficult to use. However, MCMC presents the worst performance and therefore we rank it as number four. In this case, MCMC is not available as an optimization library and requires to be implemented. The results are fair and it is the fastest of all the tools, but it is difficult to set the correct number of iterations to avoid over-fitting.

\section{Conclusions}

In this paper, we have presented a comparative study of four relevant hyper-parameter optimization approaches in the context of MOT system. The tools are reviewed with respect to performance criteria, accessibility, computational cost time, among others. To the best of our knowledge, they are not comparative studies of optimization tools that study the tuning influence over tracking systems. This application is an example but can be extended to others.

We have shown how the same application can provide better results by using a better combination of parameters, and how these can be found using expert tools. Our goal is to introduce the reader these four optimization methods, with their respective tools, and motivate their use with respect to a criterion provided in the Tab. 9. Thus, new methods that use optimized hyper parameters will give results that reflect their maximum potential. We have highlighted the strengths and weaknesses of each as detailed as possible considering many criteria.

\section{References}

1. Berclaz, J., Turetken, E., Fleuret, F., Fua, P.: Multiple object tracking using k-shortest paths optimization. IEEE Trans. on Pattern Analysis and Machine Intelligence (2011)

2. Bergstra, J.S., , Bardenet, R., , Bengio, Y., Kégl, B.: Algorithms for hyper-parameter optimization. In: J. Shawe-Taylor, R.S. Zemel, P.L. Bartlett, F. Pereira, K.Q. Weinberger (eds.) Advances in Neural Information Processing Systems, vol. 24, pp. 2546-2554 (2011)

3. Bergstra, J.S., Bardenet, R., Bengio, Y., Kégl, B.: Algorithms for hyper-parameter optimization. In: J. Shawe-Taylor, R.S. Zemel, P.L. Bartlett, F. Pereira, K.Q. Weinberger (eds.) Advances in Neural Information Processing Systems 24, pp. 2546-2554. Curran Associates, Inc. (2011)

4. Bernardin, K., Stiefelhagen, R.: Evaluating multiple object tracking performance: the clear mot metrics. EURASIP Journal on Image and Video Processing 2008(1), 1-10 (2008)

5. Bewley, A., Ge, Z., Ott, L., Ramos, F., Upcroft, B.: Simple online and realtime tracking. In: 2016 IEEE International Conference on Image Processing (ICIP), pp. 3464-3468 (2016). DOI 10.1109/ICIP.2016.7533003

6. Binitha, S., Sathya, S.: A survey of bio inspired optimization algorithms 2, 137-151 (2012)

7. Bishop, C.M.: Pattern Recognition and Machine Learning (Information Science and Statistics). Springer-Verlag New York, Inc., Secaucus, NJ, USA (2006)

8. Boussad, I., Lepagnot, J., Siarry, P.: A survey on optimization metaheuristics. Information Sciences 237, 82 - 117 (2013). Prediction, Control and Diagnosis using Advanced Neural Computations
9. Breitenstein, M.D., Reichlin, F., Leibe, B., Koller-Meier, E., Van Gool, L.: Online multiperson tracking-by-detection from a single, uncalibrated camera. IEEE Trans. on Pattern Analysis and Machine Intelligence 33(9), 1820-1833 (2011)

10. Burgard, W., Brock, O., Stachniss, C.: Active Policy Learning for Robot Planning and Exploration under Uncertainty, pp. 352-. MIT Press (2008). URL $\mathrm{http}: / /$ ieeexplore.ieee.org/xpl/articleDetails.jsp?arnumber $=6280104$

11. Chodorow, K., Dirolf, M.: MongoDB: The Definitive Guide, 1st edn. O'Reilly Media, Inc. (2010)

12. Dollr, P., Appel, R., Belongie, S., Perona, P.: Fast feature pyramids for object detection. IEEE Transactions on Pattern Analysis and Machine Intelligence 36(8), 1532-1545 (2014). DOI 10.1109/TPAMI.2014.2300479

13. Domhan, T., Springenberg, J.T., Hutter, F.: Speeding up automatic hyperparameter optimization of deep neural networks by extrapolation of learning curves. In: Proceedings of the 24th International Conference on Artificial Intelligence, IJCAI'15, pp. 3460-3468. AAAI Press (2015). URL http://dl.acm.org/citation.cfm?id=2832581.2832731

14. Ess, A., Leibe, B., Gool, L.V.: Depth and appearance for mobile scene analysis. In: 2007 IEEE 11th International Conference on Computer Vision, pp. 1-8 (2007). DOI 10.1109/ICCV.2007.4409092

15. Ferryman, J., Shahrokni, A.: Pets2009: Dataset and challenge. In: Twelfth IEEE Int. Workshop on Performance Evaluation of Tracking and Surveillance, pp. 1-6 (2009). DOI 10.1109/PETSWINTER.2009.5399556

16. Floudas, C.A., Gounaris, C.E.: A review of recent advances in global optimization. Journal of Global Optimization 45(1), 3 (2008). DOI 10.1007/s10898-008-9332-8. URL https://doi.org/10.1007/s10898-008-9332-8

17. Guo, L.: Stability of recursive stochastic tracking algorithms. SIAM Journal on Control and Optimization 32(5), 11951225 (1994). DOI $10.1137 / \mathrm{S} 0363012992225606 . \quad$ URL https://doi.org/10.1137/S0363012992225606

18. Haftka, R.T., Villanueva, D., Chaudhuri, A.: Parallel surrogateassisted global optimization with expensive functions - a survey. Structural and Multidisciplinary Optimization 54(1), 3-13 (2016). DOI 10.1007/s00158-016-1432-3. URL https://doi.org/10.1007/s00158-016-1432-3

19. Hutter, F., , Hoos, H., Leyton-Brown, K.: Sequential model-based optimization for general algorithm configuration. In: Proc. of LION-5, pp. 507-523 (2011)

20. Hutter, F., , Hoos, H., Leyton-Brown, K.: An efficient approach for assessing hyperparameter importance. In: Proceedings of the 31 st International Conference on International Conference on Machine Learning, ICML'14, vol. 32, pp. I-754-I-762 (2014)

21. Kim, K., Davis, L.: Multi-camera tracking and segmentation of occluded people on ground plane using search-guided particle filtering. Computer Vision-ECCV 2006 pp. 98-109 (2006)

22. Leal-Taixé, L., Milan, A., Reid, I., Roth, S., Schindler, K.: MOTChallenge 2015: Towards a benchmark for multi-target tracking (2015). URL http://arxiv.org/abs/1504.01942

23. Lizotte, D., Wang, T., Bowling, M., Schuurmans, D.: Automatic gait optimization with gaussian process regression. In: Proceedings of the 20th International Joint Conference on Artifical Intelligence, IJCAI'07, pp. 944-949. Morgan Kaufmann Publishers Inc., San Francisco, CA, USA (2007). URL http://dl.acm.org/citation.cfm?id=1625275.1625428

24. Luo, G.: A review of automatic selection methods for machine learning algorithms and hyper-parameter values. Network Modeling Analysis in Health Informatics and Bioinformatics 5(1), 18 (2016). DOI 10.1007/s13721-016-0125-6. URL https://doi.org/10.1007/s13721-016-0125-6

25. Luo, W., Zhao, X., Kim, T.: Multiple object tracking: A review. CoRR abs/1409.7618 (2014). URL http://arxiv.org/abs/1409.7618 
26. Maggio, E., Taj, M., Cavallaro, A.: Efficient multitarget visual tracking using random finite sets. IEEE Transactions on Circuits and Systems for Video Technology 18(8), 1016-1027 (2008). DOI 10.1109/TCSVT.2008.928221

27. Maurice, C., Madrigal, F., Lerasle, F.: Hyper-optimization tools comparison for parameter tuning applications. In: 2017 14th IEEE International Conference on Advanced Video and Signal Based Surveillance (AVSS), pp. 1-6 (2017). DOI 10.1109/AVSS.2017.8078499

28. Minton, S., Johnston, M.D., Philips, A.B., Laird, P.: Minimizing conflicts: a heuristic repair method for constraint satisfaction and scheduling problems. Artificial Intelligence 58(1-3), 161-205 (1992)

29. Nocedal, J., Wright, S.J.: Numerical Optimization, second edition. World Scientific (2006)

30. Pazhaniraja, N., Paul, P.V., Roja, G., Shanmugapriya, K., Sonali, B.: A study on recent bio-inspired optimization algorithms. In: 2017 Fourth International Conference on Signal Processing, Communication and Networking (ICSCN), pp. 1-6 (2017). DOI 10.1109/ICSCN.2017.8085674

31. Qin, Z., Shelton, C.R.: Improving multi-target tracking via social grouping. In: 2012 IEEE Conference on Computer Vision and Pattern Recognition, pp. 1972-1978 (2012). DOI 10.1109/CVPR.2012.6247899

32. Shahriari, B., Swersky, K., Wang, Z., Adams, R.P., de Freitas, N.: Taking the human out of the loop: A review of bayesian optimization. Proceedings of the IEEE 104(1), 148-175 (2016). DOI 10.1109/JPROC.2015.2494218

33. Shan, S., Wang, G.G.: Survey of modeling and optimization strategies to solve high-dimensional design problems with computationally-expensive black-box functions. Structural and Multidisciplinary Optimization 41(2), 219-241 (2010). DOI 10.1007/s00158-009-0420-2

34. Smeulders, A.W.M., Chu, D.M., Cucchiara, R., Calderara, S., Dehghan, A., Shah, M.: Visual tracking: An experimental survey. IEEE Transactions on Pattern Analysis and Machine Intelligence 36(7), 1442-1468 (2014). DOI 10.1109/TPAMI.2013.230

35. Snoek, J., Larochelle, H., Adams, R.P.: Practical bayesian optimization of machine learning algorithms. In: Proceedings of the 25th International Conference on Neural Information Processing Systems - Volume 2, NIPS'12, pp. 2951-2959. Curran Associates Inc., USA (2012)

36. Snoek, J., Rippel, O., Swersky, K., Kiros, R., Satish, N., Sundaram, N., Patwary, M., Prabhat, M., Adams, R.: Scalable bayesian optimization using deep neural networks. In: F. Bach, D. Blei (eds.) Proceedings of the 32nd International Conference on Machine Learning, Proceedings of Machine Learning Research, vol. 37, pp. 2171-2180. PMLR, Lille, France (2015). URL http://proceedings.mlr.press/v37/snoek15.html

37. Tsamardinos, I., Rakhshani, A., Lagani, V.: Performanceestimation properties of cross-validation-based protocols with simultaneous hyper-parameter optimization. In: A. Likas, K. Blekas, D. Kalles (eds.) Artificial Intelligence: Methods and Applications, pp. 1-14. Springer International Publishing, Cham (2014)

38. Wang, Z., Zoghi, M., Hutter, F., Matheson, D., de Freitas, N.: Bayesian optimization in high dimensions via random embeddings. In: International Joint Conferences on Artificial Intelligence (IJCAI) - Distinguished Paper Award (2013). URL http://www.cs.ubc.ca/ hutter/papers/13-IJCAI-BO-highdim.pdf

39. Watada, J., Musa, Z., Jain, L.C., Fulcher, J.: Human tracking: A state-of-art survey. In: R. Setchi, I. Jordanov, R.J. Howlett, L.C. Jain (eds.) Knowledge-Based and Intelligent Information and Engineering Systems, pp. 454-463. Springer Berlin Heidelberg, Berlin, Heidelberg (2010)
40. Weise, T.: Global Optimization Algorithms - Theory and Application, second edn. Self-Published (2009). URL http://www.itweise.de/. Online available at http://www.it-weise.de/

41. Wu, Y., Lim, J., Yang, M.H.: Object tracking benchmark. IEEE Transactions on Pattern Analysis and Machine Intelligence 37(9), 1834-1848 (2015). DOI 10.1109/TPAMI.2014.2388226 